\title{
Membranes of Polyvinylidene Fluoride and PVDF Nanocomposites with Carbon Nanotubes via Immersion Precipitation
}

\author{
Gaurav Mago, ${ }^{1}$ Dilhan M. Kalyon, ${ }^{2}$ and Frank T. Fisher ${ }^{1}$ \\ ${ }^{1}$ Department of Mechanical Engineering, Stevens Institute of Technology, Hoboken, NJ 07030, USA \\ ${ }^{2}$ Department of Chemical Engineering and Materials Science, Stevens Institute of Technology, Hoboken, NJ 07030, USA
}

Correspondence should be addressed to Frank T. Fisher, ffisher@stevens.edu

Received 24 September 2007; Accepted 21 March 2008

Recommended by Jun Lou

\begin{abstract}
Microporous polyvinylidene fluoride (PVDF) and PVDF nanocomposite membranes were prepared via an isothermal immersion precipitation method using two different antisolvents (ethanol and water). The structure and morphology of the resulting membranes were investigated by wide angle X-ray diffraction (WAXD), Fourier transform infrared spectroscopy (FTIR), scanning electron microscopy (SEM), and differential scanning calorimetry (DSC). The effects of the type of the antisolvent and the presence of multiwalled carbon nanotubes (MWNTs) on membrane morphology and the crystal structure developed within the membranes were studied. The crystallization of the PVDF upon immersion precipitation occurred predominantly in the $\alpha$-phase when water is used as the antisolvent or in the absence of the carbon nanotubes. On the other hand, $\beta$-phase crystallization of the PVDF was promoted upon the use of ethanol as the antisolvent in conjunction with the incorporation of the MWNTs. The morphology and the total crystallinity of the PVDF membranes were also affected by the incorporation of the MWNTs and the antisolvent used, suggesting that the microstructure and the ultimate properties of the PVDF membranes can be engineered upon the judicious selection of crystallization conditions and the use of carbon nanotubes.
\end{abstract}

Copyright (c) 2008 Gaurav Mago et al. This is an open access article distributed under the Creative Commons Attribution License, which permits unrestricted use, distribution, and reproduction in any medium, provided the original work is properly cited.

\section{INTRODUCTION}

The physical properties of semicrystalline polymers depend upon the processing conditions [1] and can be strongly influenced by the presence of nanoparticles, which affect the crystallization behavior [2] and the resulting crystal morphology developed within the processed sample. Polyvinylidene fluoride (PVDF) is a semicrystalline thermoplastic polymer with five possible polymorphs [3]. Among these polymorphs, more attention has been paid to the $\beta$-phase due to its piezoelectric, ferroelectric, and pyroelectric properties [47]. Since the increase in the $\beta$-phase of PVDF has been associated with a greater piezoelectric coefficient (due to an enhancement of the electromechanical coupling coefficient), a variety of experimental techniques have been developed to induce $\beta$-phase formation in PVDF $[8,9]$. For example, Matsushige and Takemura showed that crystallization from themelt at pressures which exceed $350 \mathrm{MPa}$ led to the formation of the $\beta$-form of PVDF [10]. Uniaxial or biaxial drawing of PVDF films has also been shown to induce an $\alpha-\beta$ transition [11-13]. In addition, a number of reports also indicate that nanoclays and carbon nanotubes can induce the $\beta$-crystal formation in PVDF nanocomposites prepared via melt processing or solution processing [14-17]. Recently, Dillon et al. used a coprecipitation method to induce the $\beta$-crystal structure for PVDF samples reinforced with nanoclays [18].

Such organic-inorganic polymer nanocomposites have attracted wide interest in the research community as researchers investigate the addition of inorganic nanoparticles to impart multifunctionality to the host polymer system, for example, simultaneous enhancement of multiple properties such as electrical and thermal conductivity, mechanical toughness, and dielectric constants of composites [19-24]. Such nanocomposites can also prove to be useful for molecular separations. For example, Merkel et al. found that physical dispersion of nonporous, nanoscale, fumed silica particles in glassy amorphous poly(4-methyl-2-pentyne) 
enhanced both membrane permeability and selectivity for large organic molecules over small gas molecules such as hydrogen [25]. In this regard, immersion precipitation is a commonly used technique to prepare PVDF membranes for microfiltration and ultrafiltration [26-32]. Membranes from electrospun polymer nanocomposites can also be used for sensing applications [33, 34]. The incorporation of carbon nanotubes into PVDF is especially attractive because of the significant increase in the electromechanical coefficient to enable the use of the resulting nanocomposites as actuators for artificial muscles and sensors for vibration control [3537].

While immersion precipitation has been used for a number of pure polymer systems [38-44], lacking are studies of the effect of MWNTs and other nanoparticles on the morphology and crystal structure of PVDF nanocomposite membranes prepared using this technique. Here, we report the utilization of the immersion precipitation technique for the preparation of PVDF nanocomposite membranes. The effects of the incorporation of MWNTs on the crystallization behavior of PVDF was investigated when ethanol and water, respectively, were used as the antisolvent in the immersion precipitation process .

\section{EXPERIMENTAL}

\subsection{Materials}

Powdered PVDF (Kynar 741) was obtained from Arkema Inc., Philadelphia, PA, USA. As reported by the manufacturer, its weight average molecular weight is 250,000 and its density is $1.78 \mathrm{~g} / \mathrm{cm}^{3}$. The MWNTs (trade name: MWNTA-P) were purchased from Sunnano, Jiangxi, China. As reported by the manufacturer, the diameter of the MWNTs was $10-30 \mathrm{~nm}$, and the average bulk density was $1.5 \mathrm{~g} / \mathrm{cm}^{3}$. To examine the size and shape distributions of the MWNTs samples, an LEO 1550 scanning electron microscope (SEM) operated at $15 \mathrm{kV}$ was used. Figure 1 shows a typical scanning electron micrograph of MWNTs. The scale bar is $1 \mu \mathrm{m}$. The solvent used was HPLC grade (99.9\%) N,Ndimethylformamide (DMF) from Sigma-Aldrich (St. Louis, MO, USA). The antisolvents used were distilled water and ethanol (A.C.S grade 99.98\% purity from Pharmco Inc. (Brookfield, CT, USA).

\subsection{Membrane preparation}

First, the PVDF powder and the MWNTs were mixed separately with DMF. The polymer solution was prepared by heating a mixture of PVDF and solvent (1:4 in weight ratio) at $70^{\circ} \mathrm{C}$ for 2 hours. The MWNT/DMF premix was mixed using a hot plate with magnetic stirrer at $50^{\circ} \mathrm{C}$ for approximately 3 hours. The final mixture was prepared by adding the contents of the MWNT/DMF premix to the PVDF solution at $70^{\circ} \mathrm{C}$ with continuous stirring. The result was a mixture that is approximately $5 \mathrm{wt} \%$ MWNT-PVDF in DMF (from here onwards, the loading of MWNTs is in wt $\%$ unless otherwise stated). This solution was cast on a glass plate and then first dried in a vacuum oven at $50^{\circ} \mathrm{C}$

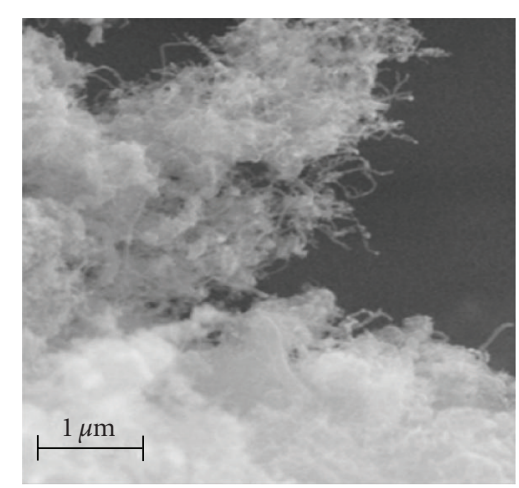

FIGURE 1: SEM micrograph of MWNTs.

for approximately 2 minutes for solvent pre-evaporation; and then the membrane was coagulated using ethanol or water antisolvents. After complete coagulation, the membranes were transferred to a vacuum oven for drying overnight at $50^{\circ} \mathrm{C}$. A similar technique was followed to prepare pure PVDF membranes for comparison purposes. Thickness of the membranes was $100-200 \mu \mathrm{m}$. The morphology of the dried membranes was examined using an LEO 1550 SEM at $20 \mathrm{kV}$. The top surface of the membranes was sputter-coated with gold before analysis.

\subsection{Membrane characterization}

Differential scanning calorimetry (DSC) studies were conducted using a TA Instruments (New Castle, DE, USA) DSC model Q1000 for pure PVDF and PVDF nanocomposite samples under a dry $\mathrm{N}_{2}$ environment. The sample weight was kept at approximately $3 \mathrm{mg}$. During DSC analysis, the precipitated and dried samples were ramped from $25^{\circ} \mathrm{C}$ to $200^{\circ} \mathrm{C}$ at a rate of $10^{\circ} \mathrm{C} / \mathrm{min}$, then maintained at isothermal conditions for 5 minutes at $200^{\circ} \mathrm{C}$. The specimens were then cooled at a rate of $10^{\circ} \mathrm{C} / \mathrm{min}$ to ambient. The nominal melting temperature $\left(T_{m, p}\right)$ was defined as the peak of the melting endotherm during first heating from 25 to $200^{\circ} \mathrm{C}$, and the nominal crystallization temperature $\left(T_{c, p}\right)$ was defined as the peak of the crystallization exotherm upon cooling from 200 to $25^{\circ} \mathrm{C}$. Wide angle X-ray diffraction (WAXD) data were collected at room temperature by positioning the membranes on a quartz sample holder using a Rigaku Miniflex diffractometer in conjunction with a $\mathrm{CuK}_{\alpha}$ radiation source $(\lambda=0.154 \mathrm{~nm})$ operated at $30 \mathrm{kV}$. The $\mathrm{X}$ ray diffractograms were collected in the scan range, $2 \theta$ of $5-50^{\circ}$ at the scan speed of $1^{\circ} \mathrm{min}^{-1}$ and using a step size of $0.04^{\circ}$. Fourier transform infrared (FTIR) spectra of the membranes were recorded on a JASCO ATR/FTIR-460 Plus over a range of $1000-550 \mathrm{~cm}^{-1}$ with a resolution of $4 \mathrm{~cm}^{-1}$.

\section{RESULTS AND DISCUSSION}

The morphology and the crystallinity of the PVDF and MWNT-PVDF nanocomposite membranes, prepared by the immersion precipitation technique, were investigated as a function of the incorporation of the MWNTs and the type 


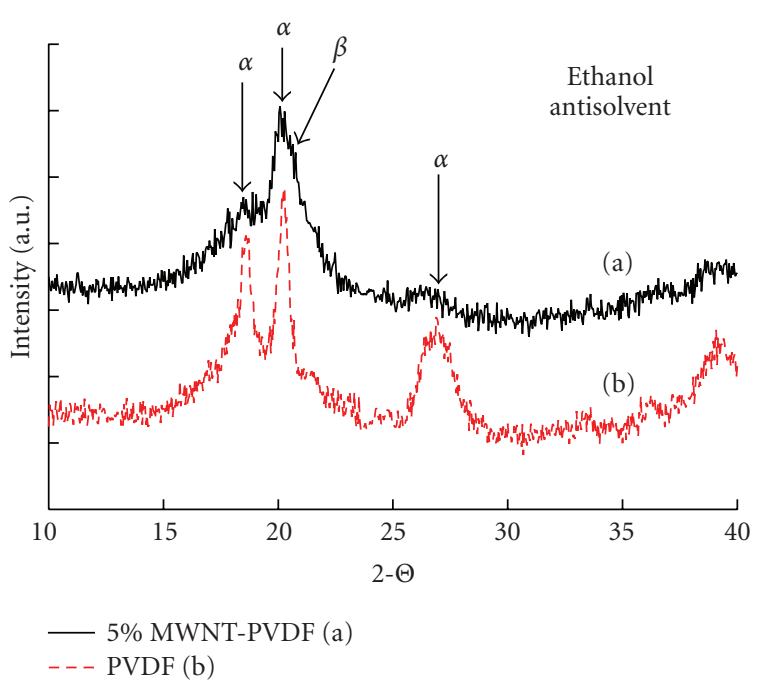

FIgURE 2: WAXD of PVDF and PVDF nanocomposite membranes prepared using ethanol as the antisolvent.

of antisolvent used. As described below, the crystallization of the PVDF upon immersion precipitation occurred predominantly in the $\alpha$-phase when water is used as the antisolvent or in the absence of the carbon nanotubes, while $\beta$-phase crystallization was favored upon the use of ethanol as the antisolvent in conjunction with the incorporation of the MWNTs.

\subsection{Effect of MWNTs and antisolvent on the PVDF crystal structure}

The results of WAXD analysis of PVDF and PVDF nanocomposite membranes prepared using ethanol as the antisolvent are shown in Figure 2. From the integration of the area under the crystalline peaks, it can be seen that the pure PVDF membrane contains predominantly major crystalline peaks at the $2 \theta$ values of $18.6^{\circ}, 20.3^{\circ}$, and $27^{\circ}$. These peaks are attributed to the crystal planes associated with the $\alpha$ phase of PVDF $[18,41]$. It is significant that the addition of $5 \%$ MWNTs into PVDF promotes the crystallization of the PVDF in the $\beta$-polymorph. This is indicated from the WAXD patterns of 5\% MWNT-PVDF nanocomposite membranes which exhibit the distinct feature of containing a sharp peak at $20.6^{\circ}$ which is attributed specifically to the presence of the $\beta$ polymorph of PVDF [14-18]. In addition to this peak, the 5\% MWNT-PVDF nanocomposite samples also exhibit large reductions in the areas under the peaks associated with the $\alpha$ polymorph that occur at the $2 \theta$ values of $18.6^{\circ}$ and $27^{\circ}$. The WAXD pattern for pure MWNTs (Figure 3 ) shows major peaks at $28.5^{\circ}, 39.5^{\circ}$, and $40.6^{\circ}$ which indicate that the crystalline peaks arising from the presence of the MWNTs are not overlapping with the crystalline peak associated with $\beta$ polymorph which occurs at $20.6^{\circ}$.

To further elucidate the effects of the concentration of the MWNTs on $\beta$-phase formation, PVDF membranes were prepared using 2 and $10 \mathrm{wt} \%$ loadings of MWNTs as shown in Figure 4. A decrease in the $\alpha$-peak area with increase in

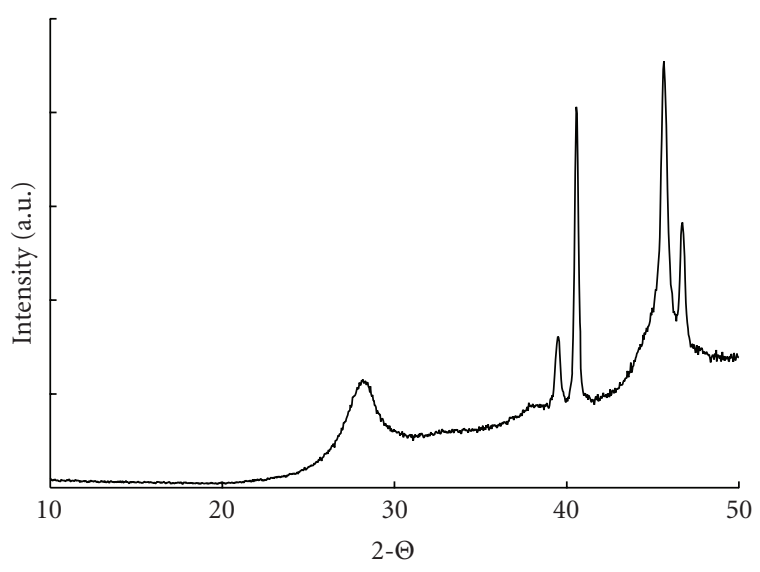

Figure 3: WAXD of pure MWNT sample.

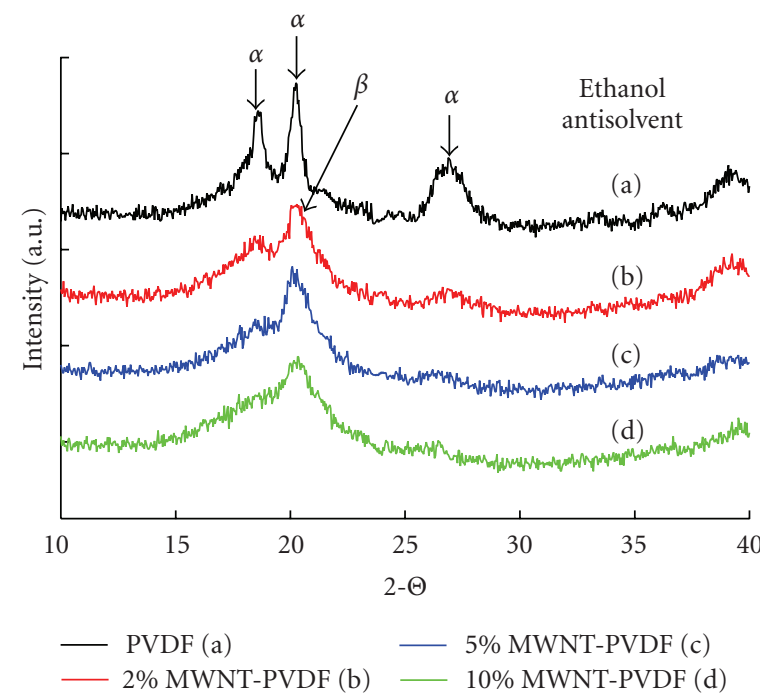

FIgURE 4: WAXD of nanocomposite membranes with different loadings of MWNTs prepared using ethanol as the antisolvent.

MWNT loadings can clearly be seen in the figure. It was found that the incorporation of MWNTs at both the 2 and $10 \mathrm{wt} \%$ levels continue to promote the crystallization of PVDF in the $\beta$-polymorph. The WAXD patterns of 2 and $10 \%$ MWNT-PVDF nanocomposites both exhibit a single sharp peak at $20.6^{\circ}$ (Figure 4). Furthermore, the effect of the polymer concentration in the premix was also investigated by using a $10 \%$ PVDF/DMF solution (compared to the 20\% PVDF solution used previously); this premix solution was then used to prepare 5\% MWNT-PVDF nanocomposite membranes using ethanol as the antisolvent. X-ray data from these samples also indicated the crystallization of the PVDF predominantly in the $\beta$-phase (not shown here). This again suggests that the $\beta$-phase formation in MWNTPVDF membranes is principally due to the presence of the nanotubes and ethanol as the antisolvent.

By comparison, the WAXD results obtained from PVDF and 5\% MWNT-PVDF membranes prepared using water as the antisolvent during the precipitation process are shown in 


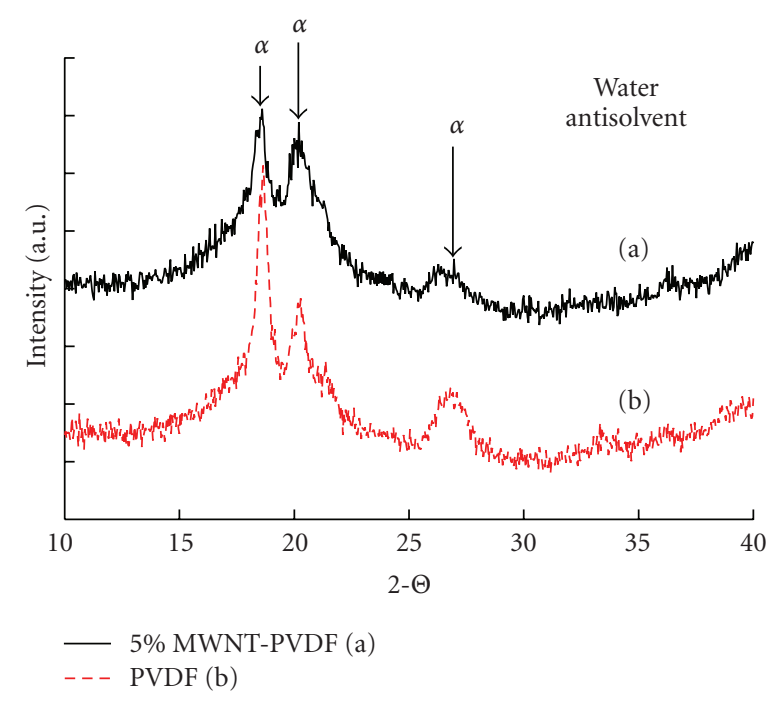

FIgURE 5: WAXD of PVDF and PVDF nanocomposite membranes prepared using water as the antisolvent.

Figure 5. Here, it can be seen that the major peaks in both membranes correspond to the $\alpha$-form of PVDF and remain largely unchanged upon the incorporation of the MWNTs. Additional WAXD results on membranes prepared using 2 and $10 \mathrm{wt} \%$ MWNTs (not shown here) show similar behavior. The difference in crystal structure attained as a function of antisolvent can be attributed to the fact that ethanol gives rise to a relatively slow rate of polymer coagulation for PVDF, whereas water as the anitsolvent results in rapid coagulation of PVDF [26, 40, 42, 45]. Upon addition of ethanol solidliquid, demixing occurs in the crystallizable segments of the polymer, which enables polymer crystallization to take place due to slow precipitation of the polymer [42]. In the case of water, the rate of polymer precipitation is very fast, leading to a relatively fast rate of crystallization, which should result in a lowered degree of crystallinity $[38,45,46]$. The principle reason that the rate of precipitation increases for the water/PVDF system should be associated with the rapid rate of interdiffusion of the solvent and the nonsolvent [45]. It is interesting to note that the nanocomposite membranes prepared using water as the antisolvent did not exhibit the $\beta$-phase (Figure 5) which can again be related to the slow coagulation rate with ethanol. Figure 3 shows that major $\alpha$ peaks in PVDF membranes are transformed to $\beta$-peak upon the incorporation of the MWNTs (5\%). As discussed further in Section 3.3, the presence of the MWNTs increases the nucleation rate for crystallization (with MWNTs acting as heterogeneous nuclei sites), giving rise to an increase in the crystallinity of the membranes versus virgin PVDF and as seen here promotes crystallization in the $\beta$ phase.

The crystal phases within the membranes were further verified using FTIR analysis. The corresponding IR absorption band characteristics of the $\alpha$-phase are 766 and $796 \mathrm{~cm}^{-1}$, whereas for $\beta$-phase peaks in the IR spectra are located at 511 and $840 \mathrm{~cm}^{-1}[20,41]$. Figure 6 shows the FTIR spectra for the pure PVDF and PVDF nanocomposite membranes prepared using ethanol and water as the anti-

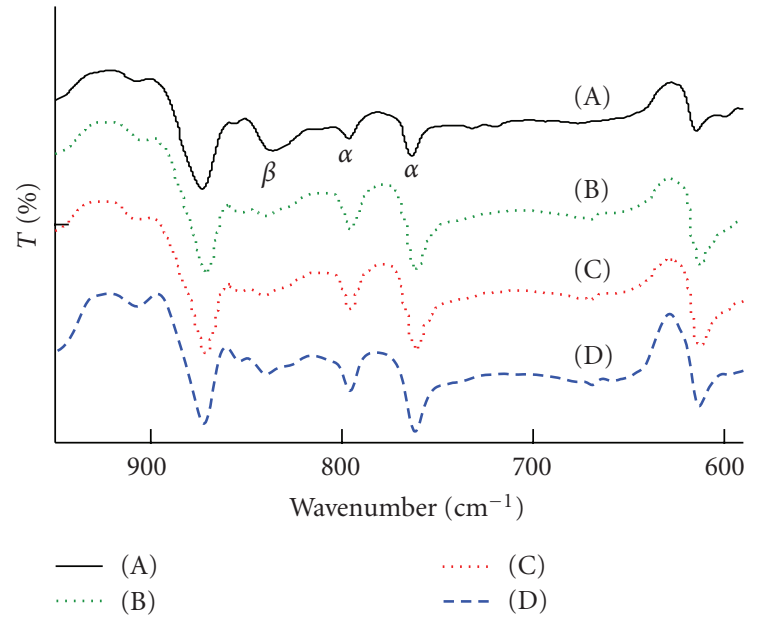

FIGURE 6: FTIR spectra of (A) 5\% MWNT-PVDF (ethanol antisolvent), (B) pure PVDF (ethanol antisolvent), (C) 5\% MWNTPVDF (water antisolvent), and (D) pure PVDF (water antisolvent) membranes.

solvent. It can be seen that 5\% MWNT-PVDF membrane prepared using ethanol antisolvent consists primarily of $\beta$ phase crystals $\left(840 \mathrm{~cm}^{-1}\right)$, along with small amount of $\alpha$ phase crystals as well (the $\alpha$-peaks at 766 and $796 \mathrm{~cm}^{-1}$ have low intensity). FTIR spectra of pure PVDF membrane prepared with ethanol antisolvent indicate predominantly the $\alpha$-crystal phase. Similarly, the FTIR results for membranes prepared with water antisolvent (both the pure PVDF and the 5\% MWNT-PVDF nanocomposite samples) are dominated by the presence of $\alpha$-crystals (peaks at 766 and $796 \mathrm{~cm}^{-1}$ ). These results along with WAXD data confirm the enhancement of $\beta$-phase crystal in 5\% MWNT-PVDF membrane prepared with ethanol antisolvent.

\subsection{Effect of MWNTs and antisolvent on morphology of PVDF membrane}

SEM images of the top surface of pure PVDF and 5\% MWNT-PVDF membranes precipitated from ethanol are shown in Figure 7. The effect of MWNTs on the membrane structure is significant and gives rise to the decrease in crystal size and to an increase of the microporosity of the PVDF membranes. From Figure 7(a), it can be seen that a sponge-like structure is formed to constitute the pure PVDF membrane. While a clear skin surface can be seen in the image, the structure of the membrane appears dominated by spherulitic crystallites in the $20-40 \mu \mathrm{m}$ diameter range.

By comparison, the incorporation of the MWNTs has a profound effect on the distributions of crystallite shapes, sizes, and porosity. The morphological features shown in Figure 7(b) suggest that MWNTs are acting as heterogeneous nucleating agents, the concentration of which determines the number of nuclei. The increase of the nucleation rate and the crystallization of the PVDF on the nanotube surfaces eliminate the impinging of the relatively large spherulitic crystallites observed with pure PVDF (see Figure 7(a)). Upon 
TABLE 1: Thermal analysis for PVDF and PVDF nanocomposite membranes.

\begin{tabular}{lcrrrrrr}
\hline Sample & Antisolvent & \multicolumn{1}{c}{$\Delta H_{m}, \mathrm{~J} / \mathrm{g}$} & \multicolumn{1}{c}{$X_{c}^{m}(\%)$} & \multicolumn{1}{c}{$T_{m, p}\left({ }^{\circ} \mathrm{C}\right)$} & \multicolumn{1}{c}{$\Delta H_{c}, \mathrm{~J} / \mathrm{g}$} & \multicolumn{1}{c}{$X_{c}^{c}(\%)$} \\
\hline PVDF & Ethanol & $51.32 \pm 1.01$ & $48.77 \pm 0.59$ & $168.03 \pm 0.39$ & $41.5 \pm 0.56$ & $39.67 \pm 0.54$ & $132.4 \pm 0.78$ \\
5\% MWNT-PVDF & Ethanol & $56.6 \pm 1.62$ & $54.41 \pm 1.24$ & $166.13 \pm 0.62$ & $41.33 \pm 2.11$ & $39.27 \pm 1.89$ & $142.89 \pm 3.02$ \\
PVDF & Water & $44.9 \pm 1.06$ & $42.9 \pm 1.05$ & $168.03 \pm 0.30$ & $41.16 \pm 1.05$ & $39.26 \pm 0.99$ & $133.48 \pm 0.39$ \\
$5 \%$ MWNT-PVDF & Water & $41.93 \pm 1.52$ & $40.09 \pm 1.45$ & $165.41 \pm 0.98$ & $38.35 \pm 2.27$ & $36.67 \pm 2.17$ & $136.41 \pm 0.50$ \\
\hline
\end{tabular}

$T_{m, p}=$ nominal melting temperature, $T_{c, p}=$ nominal crystallization temperature, $\Delta H_{m}$ = heat of fusion, $\Delta H_{c}=$ heat of crystallization, $X_{c}^{m}=$ crystallinity from melting endotherm, $X_{c}^{c}=$ crystallinity from crystallization exotherm. Average values and $95 \%$ confidence intervals are reported.

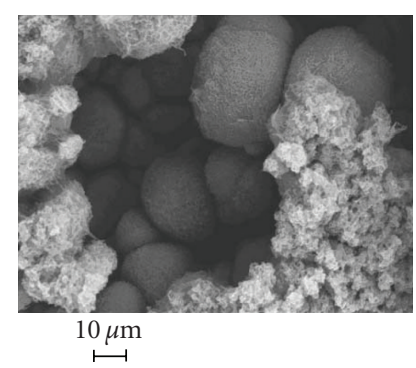

(a) PVDF

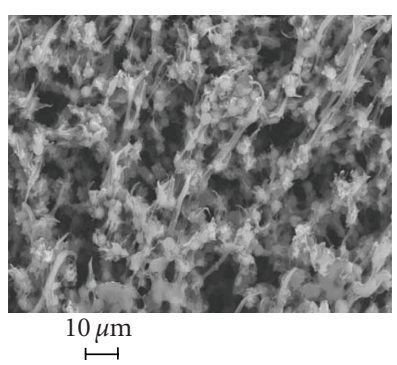

(b) $5 \%$ MWNT-PVDF
Figure 7: SEM of (a) PVDF and (b) 5\% MWNT-PVDF nanocomposite membranes prepared using ethanol as the antisolvent.

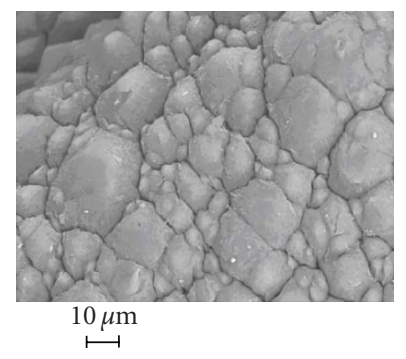

(a) PVDF

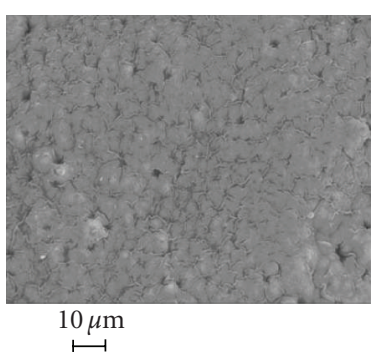

(b) 5\% MWNT-PVDF
FIgURE 8: SEM images of (a) PVDF and (b) 5\% MWNT-PVDF nanocomposite membranes prepared using water as the antisolvent.

the incorporation of the MWNTs, a porous superstructure consisting of shish-kebab-like crystalline entities, forming a loose network, is observed. It also appears that the nanotubes are providing interconnections to form a continuous and open structure. The interconnectivity of the membrane will likely provide additional mechanical strength to the membrane, possibly increasing the utility of such piezoelectric membranes.

On the other hand, Figure 8 shows the PVDF and 5\% MWNT-PVDF membranes prepared using water as the antisolvent. The top surface of the PVDF membrane shows a dense "skin" layer, which appears to be nonporous [38, 45]. The skin is composed of intersecting polygonal plates with clear boundaries; similar structures with crystalline polygonal plates have been observed in Nylon- 6 membranes prepared via the immersion precipitation in water $[39,46]$. As noted earlier, the formation of the skin layer and lack of an interconnected pore structure upon the use of water as the antisolvent is likely due to the rapid precipitation that occurs when water is used as the antisolvent, associated with the high rate of interdiffusion of DMF and water [45], where the rate of interdiffusion depends on the value of the solubility parameters of the solvent and antisolvent. In addition, small micropores can be seen on the surface of the membrane. Similar effects on morphology have been observed in micaintercalated Nylon-6 nanocomposite membrane prepared by phase inversion method using water as the antisolvent [39]. As shown in Figure 8(b), the incorporation of MWNTs results in a similar morphology, albeit with smaller polygonal regions making up the surface of the membrane.

These initial results, together with the WAXD results presented in Section 3.1, indicate that the overall crystal morphology and porosity of the PVDF nanocomposite membranes are strongly influenced by both the antisolvent used to prepare the membrane as well as the presence of the MWNTs. Further analysis of the effects of these parameters on the crystallinity, porosity, and the availability of the targeted $\beta$ crystalline phase in PVDF nanocomposite membranes is necessary to allow the optimization of the microstructure and associated ultimate properties of such membranes.

\subsection{Effects of MWNTs and antisolvent on melting and crystallization behavior of PVDF membrane}

DSC analysis of pure PVDF and PVDF nanocomposite membranes was conducted to examine the effect of MWNTs and antisolvents on the degree of crystallinity $X_{c}$ of the membranes upon precipitation and the melting/crystallization temperatures observed upon the heating and the subsequent cooling of the membranes. Representative DSC heating and cooling curves are shown in Figures 9 and 10, respectively, while the results for four samples tested at each condition are summarized in Table 1 . The heat of fusion $\left(\Delta H_{m}\right)$ data obtained for nanocomposite samples was corrected due to the presence of MWNTs in the sample (such that $\Delta H_{\text {corrected }}=\Delta H_{\text {measured }} / W_{p}$, where $W_{p}$ is the weight fraction of polymer in the sample). Similarly, the heat of crystallization $\left(\Delta H_{c}\right)$ data for PVDF nanocomposite samples was also corrected for true heat of crystallization of PVDF.

There are significant differences in the heat of fusion values for the four cases (with and without the MWNTs, and with ethanol or water). The use of ethanol as the antisolvent versus water gives rise to a greater heat of fusion as shown in Table 1. The percent crystallinity of PVDF and PVDF nanocomposites, based on either the melting $\left(X_{c}^{m}\right)$ 


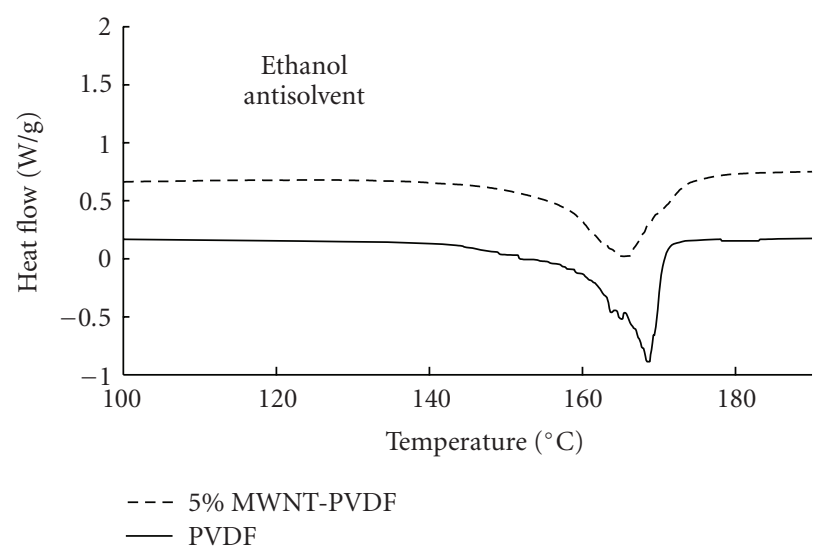

(a)

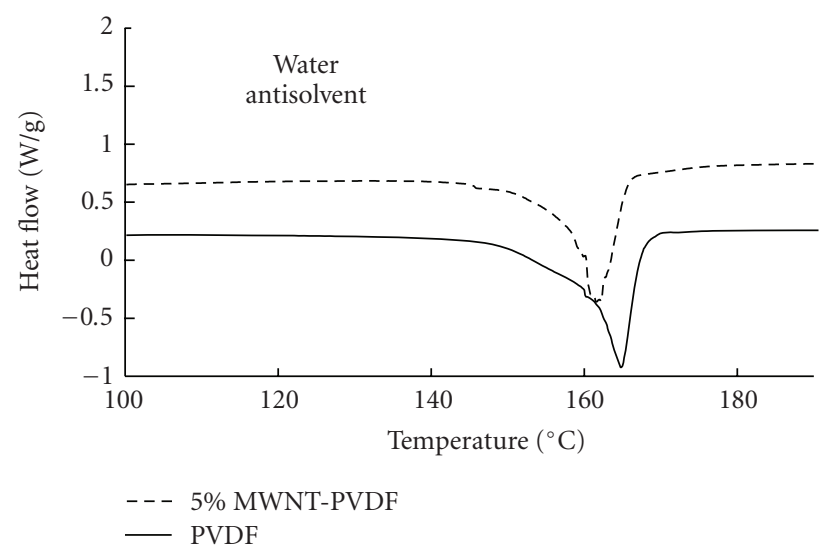

(b)

FIGURE 9: DSC endotherms of PVDF and 5\% MWNT-PVDF nanocomposite membranes scanned at $10^{\circ} \mathrm{C} / \mathrm{min}$ (a) using ethanol as the antisolvent and (b) using water as the antisolvent.

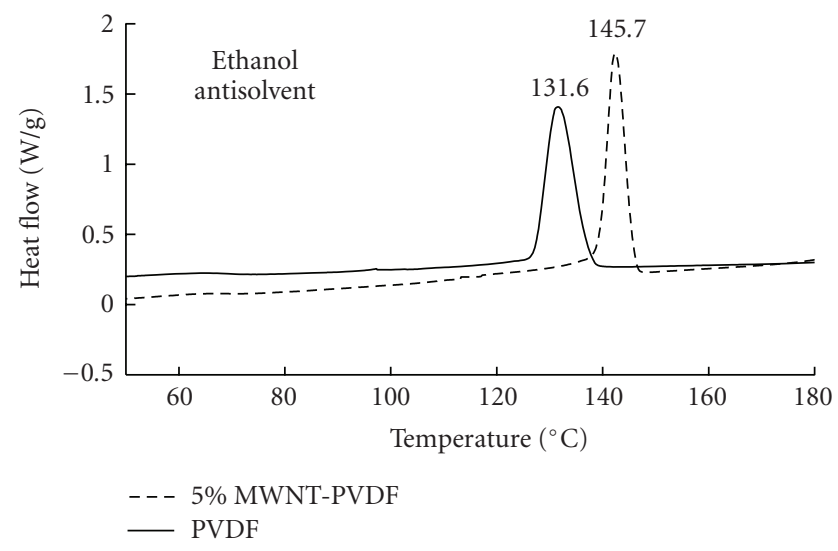

(a)

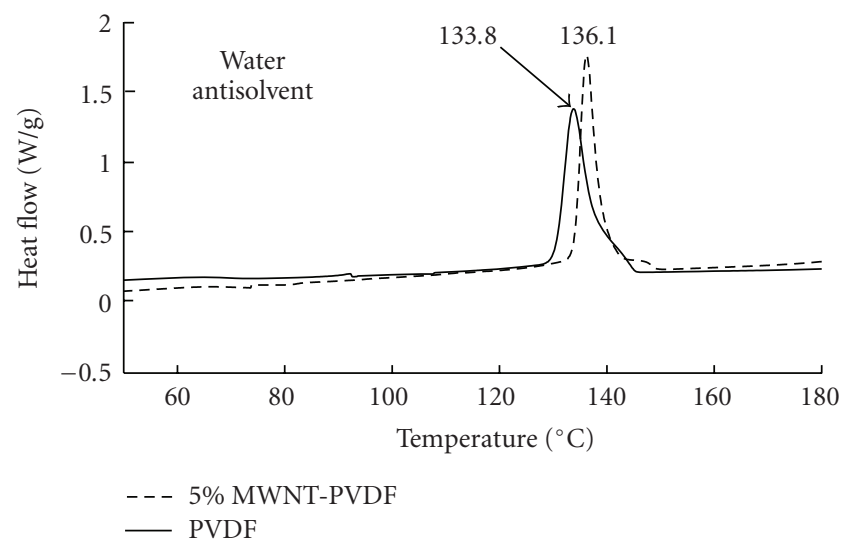

(b)

FIGURE 10: DSC exotherms for PVDF and 5\% MWNT-PVDF nanocomposite membrane scanned at $10^{\circ} \mathrm{C} / \mathrm{min}$ (a) using ethanol antisolvent and (b) using water antisolvent.

or crystallization $\left(X_{c}^{c}\right)$ DSC scans, can be obtained from the expressions:

$$
X_{c}^{m}=\frac{\Delta H_{m} * 100}{\Delta H_{100 \%, \text { crystalline }}}, \quad X_{c}^{c}=\frac{\Delta H_{c} * 100}{\Delta H_{100 \%, \text { crystalline }}},
$$

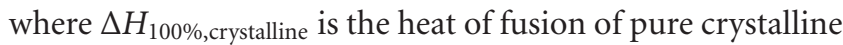
PVDF, which is reported to be $104.6 \mathrm{~J} / \mathrm{g}$ [14], and $\Delta H_{m}$ and $\Delta H_{c}$ are the heat of fusion and the heat of crystallization of the sample. As shown in Table 1, the degree of crystallinity $X_{c}^{m}$ of the PVDF is greater when ethanol is used as the antisolvent. As indicated earlier, experimental studies of interdiffusion rates of ethanol and water in similar solvents have suggested that the interdiffusion rate of water in the solvent would be significantly higher than that of ethanol [45], giving rise to a significantly higher rate of precipitation in water and thus restricting the degree of crystallinity of PVDF.

The role played by the nanotubes in the crystallization process is interesting and again depends on which antisolvent is utilized during the precipitation. With ethanol, the favorable relatively slow rate of precipitation allows the MWNTs to act as nucleating agents to induce an increase in the nucleation and enables crystallization to take place to a greater extent. The presence of the MWNTs further increases the degree of the crystallinity $X_{c}^{m}$ of PVDF (Table 1), which is consistent with the SEM micrographs showing a decrease in the size of the crystallites upon the incorporation of the MWNTs (Figure 7(b)). On the other hand, the MWNTs incorporated into the PVDF membranes upon using water as the antisolvent have no favorable effect on the crystallization of PVDF and are even detrimental, that is, a reduction in the heat of fusion and hence the degree of crystallinity is observed. It appears that during the rapid precipitation process associated with water as the antisolvent, the MWNTs presumably remain as clusters and immobilize some of the PVDF, preventing their crystallization and thus reducing the degree of crystallinity $X_{c}^{m}$ of PVDF upon precipitation.

The data associated with the crystallization peak temperature further reinforce this understanding associated with 
the roles of the rate of precipitation and the effects of the MWNTs (Table 1). The nominal crystallization temperature $\left(T_{c, p}\right)$ was found to increase upon the addition of MWNTs, which is attributed to the heterogeneous nucleation induced by the presence of the MWNTs and the associated increase in the rate of nucleation/crystallization [47]. The increase in $T_{c, p}$ is significantly higher in the case of nanocomposite membranes prepared using ethanol. In contrast, a small change in crystallization temperature and a comparatively small decrease in polymer crystal size in nanocomposite membranes are observed when water is used as the antisolvent. In addition, little change is observed in the percent crystallinity $X_{c}^{c}$ based on the DSC cooling scans.

\section{CONCLUSIONS}

MWNT-PVDF nanocomposite membranes were prepared using ethanol and water antisolvents. The desirable $\beta$-phase crystallization of PVDF was promoted using MWNTs and ethanol as the antisolvent. The crystal size of the polymer was found to decrease due to addition of MWNTs, using ethanol, while the degree of crystallinity and the crystallization temperature increased. Further tests to correlate the mechanical and piezoelectric properties of the membranes as a function of the processing parameters are ongoing. These results indicate that the microstructure and the ultimate properties of the PVDF membranes can be engineered upon the judicious selection of crystallization conditions and the use of carbon nanotubes, with applications in the engineering of surfaces and structures for targeted applications such as sensors, filters, and artificial muscles.

\section{ACKNOWLEDGMENTS}

Financial support from the Department of Mechanical Engineering at Stevens (for GM) is greatly appreciated. The authors would like to thank Dr. Halil Gevgilili from the Highly Filled Materials Institute (HfMI) at Stevens for his contributions to this work. Arkema Inc. (USA) is acknowledged for providing Kynar 741 PVDF powder. We also thank Dr. Jim Quinn, SUNY Stony Brook, for his help with the SEM imaging.

\section{REFERENCES}

[1] M. R. Kamal, D. M. Kalyon, and J. Dealy, "An integrated experimental study of the injection molding behavior of some polyethylene resins," Polymer Engineering \& Science, vol. 20, no. 17 , pp. $1117-1125,1980$.

[2] G. Mago, F. T. Fisher, and D. M. Kalyon, "Effect of shearing on the crystallization behavior of poly(butylene terephthalate) and PBT nanocomposites," in Proceedings of the ASME International Mechanical Engineering Congress and Exposition (IMECE '06), Chicago, Ill, USA, November 2006.

[3] A. J. Lovinger, "Ferroelectric polymers," Science, vol. 220, no. 4602, pp. 1115-1121, 1983.

[4] X. He, K. Yao, and B. K. Gan, "Phase transition and properties of a ferroelectric poly(vinylidene fluoridehexafluoropropylene) copolymer," Journal of Applied Physics, vol. 97, no. 8, Article ID 084101, 6 pages, 2005.
[5] A. Ambrosy and K. Holdik, "Piezoelectric PVDF films as ultrasonic transducers," Journal of Physics E, vol. 17, no. 10, pp. 856-859, 1984.

[6] M. G. Broadhurst and G. T. Davis, "Physical basis for piezoelectricity in PVDF," Ferroelectrics, vol. 60, pp. 3-13, 1984.

[7] A. J. Lovinger, "Crystallization of the $\beta$ phase of poly(vinylidene fluoride) from the melt," Polymer, vol. 22, no. 3, pp. 412-413, 1981.

[8] J. Scheinbeim, C. Nakafuku, B. A. Newman, and K. D. Pae, "High-pressure crystallization of poly(vinylidene fluoride)," Journal of Applied Physics, vol. 50, no. 6, pp. 4399-4405, 1979.

[9] G. T. Davis, J. E. McKinney, M. G. Broadhurst, and S. C. Roth, "Electric-field-induced phase changes in poly(vinylidene fluoride)," Journal of Applied Physics, vol. 49, no. 10, pp. 49985002, 1978.

[10] K. Matsushige and T. Takemura, "Melting and crystallization of poly(vinylidene fluoride) under high pressure," Journal of Polymer Science B, vol. 16, no. 5, pp. 921-934, 1978.

[11] B. Mohajir and N. Heymans, "Changes in structural and mechanical behavior of PVDF with processing or thermal treatment-2: evolution of mechanical behavior," Polymer, vol. 42, no. 16, pp. 7017-7023, 2001.

[12] B. Mohajir and N. Heymans, "Changes in structural and mechanical behavior of PVDF with processing and thermomechanical treatments-1: change in structure," Polymer, vol. 42, no. 13, pp. 5661-5667, 2001.

[13] C.-H. Du, B.-K. Zhu, and Y.-Y. Xu, "Effects of stretching on crystalline phase structure and morphology of hard elastic PVDF fibers," Journal of Applied Polymer Science, vol. 104, no. 4, pp. 2254-2259, 2007.

[14] J. Buckley, P. Cebe, D. Cherdack, et al., "Nanocomposites of poly(vinylidene fluoride) with organically modified silicate," Polymer, vol. 47, no. 7, pp. 2411-2422, 2006.

[15] N. Levi, R. Czerw, S. Xing, P. Iyer, and D. L. Carroll, "Properties of polyvinylidene difluoride-carbon nanotube blends," Nano Letters, vol. 4, no. 7, pp. 1267-1271, 2004.

[16] Y. W. Nam, W. N. Kim, Y. H. Cho, et al., "Morphology and physical properties of binary blend based on PVDF and multiwalled carbon nanotube," Macromolecular Symposia, vol. 249250, no. 1, pp. 478-484, 2007.

[17] L. Priya and J. P. Jog, "Polymorphism in intercalated poly(vinylidene fluoride)/clay nanocomposites," Journal of Applied Polymer Science, vol. 89, no. 8, pp. 2036-2040, 2003.

[18] D. R. Dillon, K. K. Tenneti, C. Y. Li, F. K. Ko, I. Sics, and B. S. Hsiao, "On the structure and morphology of polyvinylidene fluoride-nanoclay nanocomposites," Polymer, vol. 47, no. 5, pp. 1678-1688, 2006.

[19] Z.-M. Dang, S.-H. Yao, and H.-P. Xu, "Effect of tensile strain on morphology and dielectric property in nanotube/polymer nanocomposites," Applied Physics Letters, vol. 90, no. 1, Article ID 012907, 3 pages, 2007.

[20] D. Shah, P. Maiti, E. Gunn, et al., "Dramatic enhancements in toughness of polyvinylidene fluoride nanocomposites via nanoclay-directed crystal structure and morphology," Advanced Materials, vol. 16, no. 14, pp. 1173-1177, 2004.

[21] D. Shah, P. Maiti, D. D. Jiang, C. A. Batt, and E. P. Giannelis, "Effect of nanoparticle mobility on toughness of polymer nanocomposites," Advanced Materials, vol. 17, no. 5, pp. 525528, 2005.

[22] Z.-M. Dang, Y.-H. Lin, and C.-W. Nan, "Novel ferroelectric polymer composites with high dielectric constants," Advanced Materials, vol. 15, no. 19, pp. 1625-1629, 2003. 
[23] M. Wang, J. Shi, K. P. Pramoda, and S. H. Goh, "Microstructure, crystallization and dynamic mechanical behavior of poly(vinylidene fluoride) composites containing poly(methyl methacrylate)-grafted multiwalled carbon nanotubes," Nanotechnology, vol. 18, Article ID 235701, 7 pages, 2007.

[24] K. Peng, L. Hornbergera, C. V. Nguyena, and U. Sopory, "Processing of dispersed and aligned multi-walled carbon nanotubes in polyvinylidene fluoride," Journal of Advanced Materials, vol. 39, no. 2, pp. 24-31, 2007.

[25] T. C. Merkel, B. D. Freeman, R. J. Spontak, et al., "Ultrapermeable, reverse-selective nanocomposite membranes," Science, vol. 296, no. 5567, pp. 519-522, 2002.

[26] S. P. Deshmukh and K. Li, "Effect of ethanol composition in water coagulation bath on morphology of PVDF hollow fibre membranes," Journal of Membrane Science, vol. 150, no. 1, pp. 75-85, 1998.

[27] K.-Y. Lin, D.-M. Wang, and J.-Y. Lai, "Nonsolvent-induced gelation and its effect on membrane morphology," Macromolecules, vol. 35, no. 17, pp. 6697-6706, 2002.

[28] W.-H. Seol, Y. M. Lee, and J.-K. Park, "Enhancement of the mechanical properties of PVDF membranes by non-solvent aided morphology control," Journal of Power Sources, vol. 170, no. 1, pp. 191-195, 2007.

[29] K. Li, J. F. Kong, D. Wang, and W. K. Teo, "Tailor-made asymmetric PVDF hollow fibers for soluble gas removal," AIChE Journal, vol. 45, no. 6, pp. 1211-1219, 1999.

[30] M. Ulbricht, "Advanced functional polymer membranes," Polymer, vol. 47, no. 7, pp. 2217-2262, 2006.

[31] H. Kataoka, Y. Saito, T. Sakai, E. Quartarone, and P. Mustarelli, "Conduction mechanisms of PVDF-type gel polymer electrolytes of lithium prepared by a phase inversion process," Journal of Physical Chemistry B, vol. 104, no. 48, pp. 11460 11464, 2000.

[32] A. Akthakul, R. F. Salinaro, and A. M. Mayes, "Antifouling polymer membranes with subnanometer size selectivity," Macromolecules, vol. 37, no. 20, pp. 7663-7668, 2004.

[33] K. Laxminarayana and N. Jalili, "Functional nanotube-based textiles: pathway to next generation fabrics with enhanced sensing capabilities," Textile Research Journal, vol. 75, no. 9, pp. 670-680, 2005.

[34] C. Seoul, Y.-T. Kim, and C.-K. Baek, "Electrospinning of poly(vinylidene fluoride)/dimethyl formamide solutions with carbon nanotubes," Journal of Applied Polymer Science B, vol. 41, no. 13, pp. 1572-1577, 2003.

[35] T. Mirfakhrai, J. D. W. Madden, and R. H. Baughman, "Polymer artificial muscles," Materials Today, vol. 10, no. 4, pp. 30-38, 2007.

[36] A. Ramaratnam, N. Jalili, and H. Rajoria, "Development of a novel strain sensor using nanotube-based materials with applications to structural vibration control," in 6th International Conference on Vibration Measurements by Laser Techniques: Advances and Applications, vol. 5503 of Proceedings of SPIE, pp. 478-485, Ancona, Italy, June 2004.

[37] A. Ramaratnam and N. Jalili, "Reinforcement of piezoelectric polymers with carbon nanotubes: pathway to next-generation sensors," Journal of Intelligent Material Systems and Structures, vol. 17, no. 3, pp. 199-208, 2006.

[38] L.-P. Cheng, D.-J. Lin, C.-H. Shih, A.-H. Dwan, and C. C. Gryte, "PVDF membrane formation by diffusion-induced phase separation-morphology prediction based on phase behavior and mass transfer modeling," Journal of Polymer Science B, vol. 37, no. 16, pp. 2079-2092, 1999.
[39] L.-P. Cheng, D.-J. Lin, and K.-C. Yang, "Formation of micaintercalated-Nylon 6 nanocomposite membranes by phase inversion method," Journal of Membrane Science, vol. 172, no. 1-2, pp. 157-166, 2000.

[40] L.-P. Cheng, T.-H. Young, L. Fang, and J.-J. Gau, "Formation of particulate microporous poly(vinylidene fluoride) membranes by isothermal immersion precipitation from the 1-octanol/dimethylformamide/poly(vinylidene fluoride) system," Polymer, vol. 40, no. 9, pp. 2395-2403, 1999.

[41] M. G. Buonomenna, P. Macchi, M. Davoli, and E. Drioli, "Poly(vinylidene fluoride) membranes by phase inversion: the role the casting and coagulation conditions play in their morphology, crystalline structure and properties," European Polymer Journal, vol. 43, no. 4, pp. 1557-1572, 2007.

[42] L.-P. Cheng, "Effect of temperature on the formation of microporous PVDF membranes by precipitation from 1octanol/DMF/PVDF and water/ DMF/PVDF systems," Macromolecules, vol. 32, no. 20, pp. 6668-6674, 1999.

[43] M. L. Yeow, Y. T. Liu, and K. Li, "Isothermal phase diagrams and phase-inversion behavior of poly(vinylidene fluoride)/solvents/additives/water systems," Journal of Applied Polymer Science, vol. 90, no. 8, pp. 2150-2155, 2003.

[44] A. J. McHugh and D. C. Miller, "The dynamics of diffusion and gel growth during nonsolvent-induced phase inversion of polyethersulfone," Journal of Membrane Science, vol. 105, no. 1-2, pp. 121-136, 1995.

[45] T.-H. Young, L.-P. Cheng, D.-J. Lin, L. Fane, and W.-Y. Chuang, "Mechanisms of PVDF membrane formation by immersion-precipitation in soft (1-octanol) and harsh (water) nonsolvents," Polymer, vol. 40, no. 19, pp. 5315-5323, 1999.

[46] T.-H. Young, D.-J. Lin, J.-J. Gau, W.-Y. Chuang, and L.-P. Cheng, "Morphology of crystalline Nylon-610 membranes prepared by the immersion-precipitation process: competition between crystallization and liquid-liquid phase separation,” Polymer, vol. 40, no. 18, pp. 5011-5021, 1999.

[47] I. Pillin, S. Pimbert, and G. Levesque, "Influence of additives on the crystallization kinetics of semicrystalline polymers. II: selective polymer-additive interaction in poly(vinylidene difluoride)-poly(methylmethacrylate) blends," Polymer Engineering \& Science, vol. 42, no. 11, pp. 2193-2201, 2002. 

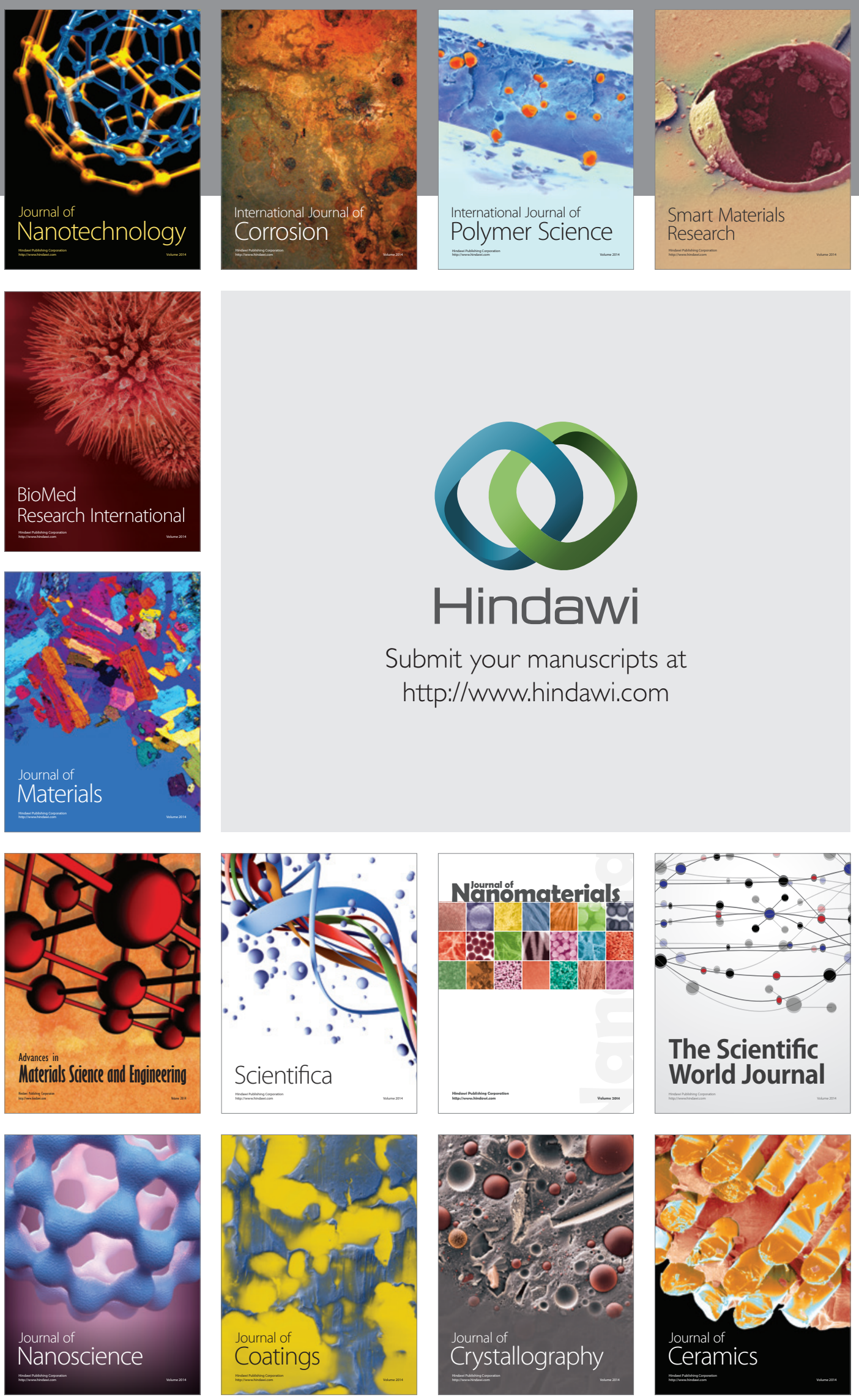

The Scientific World Journal

Submit your manuscripts at

http://www.hindawi.com

\section{World Journal}

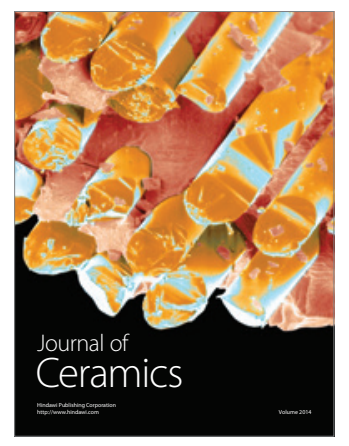

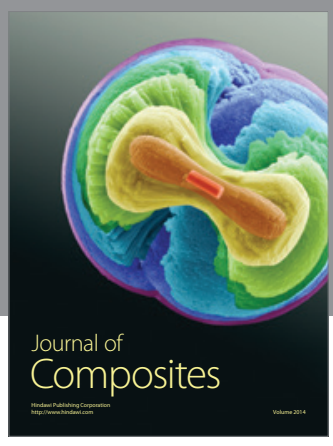
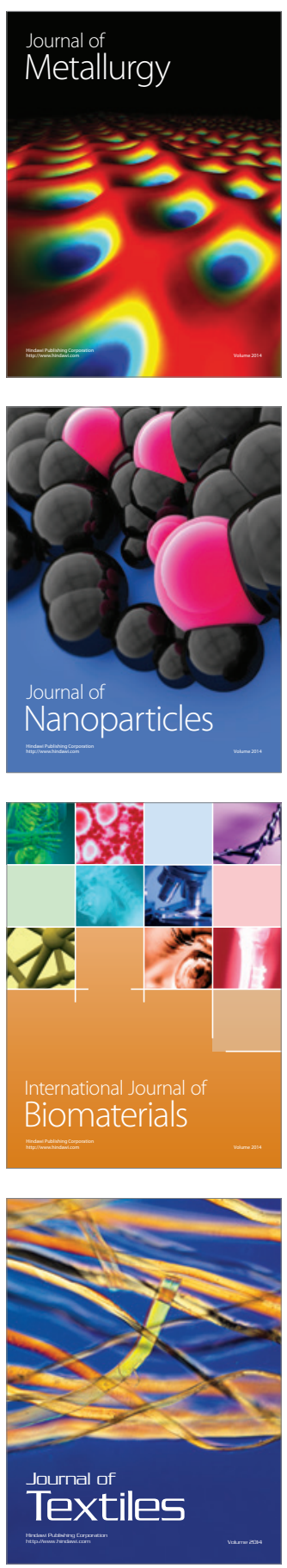